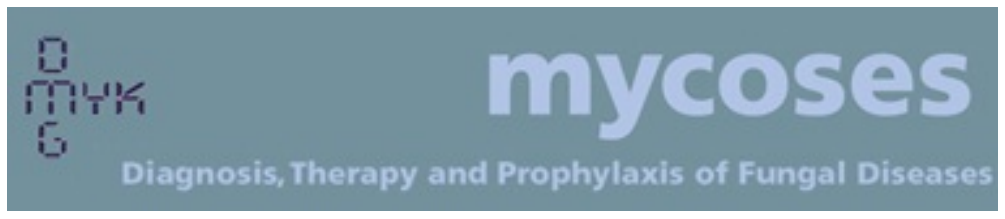

\title{
Methylene blue vs. methyl aminolevulinate photodynamic therapy in combination with oral terbinafine in the treatment of severe dermatophytic toenail onychomycosis: short- and long-term effects.
}

\begin{tabular}{|c|c|}
\hline Journal: & Mycoses \\
\hline Manuscript ID & MYC-OA-2020-154.R1 \\
\hline Manuscript Type: & Original Article \\
\hline $\begin{array}{l}\text { Date Submitted by the } \\
\text { Author: }\end{array}$ & $\mathrm{n} / \mathrm{a}$ \\
\hline Complete List of Authors: & $\begin{array}{l}\text { Alberdi, Enrique; Private clinic of Dr. Alberdi } \\
\text { Gómez, Clara; Consejo Superior de Investigaciones Cientificas }\end{array}$ \\
\hline Keywords: & antifungal agents, dermatophytes, onychomycosis, terbinafine \\
\hline Area of Expertise: & Antimycotic Therapy \\
\hline Additional Keywords: & Photodynamic therapy, methylene blue, methyl aminolevulinate \\
\hline
\end{tabular}

\section{SCHOLARONE \\ Manuscripts}


Title: Methylene blue vs. methyl aminolevulinate photodynamic therapy in combination with oral terbinafine in the treatment of severe dermatophytic toenail onychomycosis: short- and long-term effects.

Running Title: MB/PDT and MAL/PDT plus TN in onychomycosis.

Authors: Enrique Alberdi ${ }^{1} \&$ Clara Gómez ${ }^{2 *}$

${ }^{1}$ Private clinic of Dr. Alberdi, Aviador Zorita 25, 28020 Madrid

${ }^{2}$ Institute of Physical Chemistry Rocasolano, CSIC, Serrano 119, 28006 Madrid, Spain

Address of authors:

Clara Gómez (corresponding author)*:

Institute of Physical Chemistry Rocasolano

Spanish National Research Council, CSIC

C/ Serrano 119, 28006 Madrid

Tel.: +34-91-561-9400 ext.961252

Fax.: +34-91-564-2431

E-mail address: c.gomez@iqfr.csic.es

\section{ACKNOWLEDGEMENTS}

This research was supported by a grant from the Eugenio Rodríguez Pascual Foundation (Madrid, Spain) and by the Spanish Research Project MICINN (Ref.: MAT 2017-83856-C3). The authors wish to thank Dr. $\mathrm{M}^{\mathrm{a}}$ José Ortíz García for her input and suggestions while designing the study protocol and Dr. Santiago Cano for his assistance with the statistical data analysis.

\section{CONFLICT OF INTEREST}

The authors declare no conflict of interest.

\section{AUTHOR CONTRIBUTIONS}

E. Alberdi and C. Gómez designed the study protocol. E. Alberdi collected patients and collected the clinical data. C. Gómez analyzed the data and wrote the initial draft. All authors critically revised the manuscripts. 


\begin{abstract}
Background: Photodynamic therapy (PDT) kills target microorganisms via reactive oxygen species (ROS) production. PDT seems to be a good alternative treatment option for onychomycosis.
\end{abstract}

Objective: To compare the efficacy of combined therapies based on oral terbinafine (TN) plus adjunctive PDT mediated by methylene blue (MB) (TN+MB/PDT) or methyl aminolevulinate (MAL) $(\mathrm{TN}+\mathrm{MAL} / \mathrm{PDT})$ in the treatment of onychomycosis.

Methods: Twenty patients affected by severe dermatophyte onychomycosis in the nails of the big toe ( $>60 \%$ disease involvement of target nail) received oral $\mathrm{TN}$ for 12 weeks and concomitantly were randomly allocated to receive 9 sessions, separated by 2 -week intervals, of urea $(40 \%)$ plus a PDT protocol mediated by MB (TN+MB/PDT: group I) or mediated by MAL (TN+MAL/PDT: group II). Clinical and mycological efficacy was evaluated at 16, 40 and 52week follow-up.

Results: Both protocols showed a significant decrease in Onychomycosis Severity Index (OSI) scores ( $<<0.05$ ), from $24.2 \pm 4.6$ to $0.7 \pm 0.6$ (group I)) and from $18.5 \pm 10.1$ to $2.1 \pm 2.0$ (group II). No side effects or complications were reported in any of the combinations used. Mycological cure rates were significantly higher during the last third of the evaluated period of time, reaching $100 \%$ and $90 \%$ in group I and group II, respectively, at the 52-week follow-up. In both modalities, complete cure was achieved in $70 \%$ of the patients at the 52-week follow-up.

Conclusions: $\mathrm{TN}+\mathrm{MB} / \mathrm{PDT}$ and $\mathrm{TN}+\mathrm{MAL} / \mathrm{PDT}$ show similar outcomes in the treatment of toenails with severe onychomycosis. PDT is an effective method to accelerate the TN-mediated healing process.

Keywords: photodynamic therapy, methylene blue, methyl aminolevulinate, severe onychomycosis, terbinafine, onychomycosis severity index. 


\section{INTRODUCTION}

Onychomycosis is a fungal nail infection caused mainly by dermatophytes (predominantly by Trichophyton rubrum), yeasts or nondermatophyte filamentous molds (responsible for $90 \%, 5 \%$ and $3 \%$ of cases, respectively). ${ }^{1,2}$ The prevalence of onychomycosis is $2-13 \%$, and up to $14-28 \%$ among elderly patients over 60 years of age. ${ }^{3}$ Compressions and microtraumas are the main triggers of onychomycosis of the toenails. This infection is more frequent in diabetics and immunocompromised patients, as well as in those suffering from peripheral arterial diseases..$^{4,5}$

Oral therapy is the preferred treatment for onychomycosis given higher cure rates over topical therapy, being its use mandatory when the disease is severe ( $<60 \%$ of involvement). ${ }^{6}$ Antifungal agents, such as terbinafine (TN), itraconazole, fluconazole, ketoconazole and griseofulvin, combined with debridement, are the most effective treatments for onychomycosis. ${ }^{7}$ In a previous review in which mycological cure rates were assessed, ${ }^{8}$ continuous TN was found to be significantly more effective (76\%) than pulse itraconazole $(63 \%)$, griseofulvin $(60 \%)$, continuous itraconazole (59\%) and fluconazole (48\%). TN is the most frequently prescribed antifungal in North America and Europe for the treatment of onychomycosis, effective against dermatophytes and some molds. The recommended dosage for $\mathrm{TN}$ in the treatment of onychomycosis is $250 \mathrm{mg}$ /day orally for 12 weeks. ${ }^{9}$ At this final point (12 weeks) or in longer duration treatments, TN may cause gastrointestinal reactions and liver injury. ${ }^{8}$ Thus, current research on the treatment of onychomycosis is focused on finding shorter-term therapies in the form of adjunctive use of topical lacquers with better adherence and new light technologies: laser, ${ }^{10,11}$ intense pulsed light (IPL), ${ }^{12}$ photodynamic therapy (PDT). ${ }^{13,14}$

PDT is an attractive treatment option involving the activation of a photosensitizer by a defined wavelength of light in the presence of oxygen. This generates reactive oxygen species which lead to selective and highly localized destruction of abnormal cells. ${ }^{15}$ Choosing an appropriate photosensitizer (PS) improves the efficacy of this therapy..$^{16}$ Previous studies on PDT in the treatment of onychomycosis have been conducted using methylene blue (MB), ${ }^{12,17}$ 5- 
aminolevulinic acid (ALA) ${ }^{14,18}$ or methyl aminolevulinate (MAL) $)^{19,20}$ as PS, all of them reporting positive results. A comparison of two or more PS in the same study with similar patient condition could be of high interest.

Although PDT could be an adjunctive method for severe onychomycosis treatment, offering the possibility of reducing treatment duration, avoiding the adverse side effects of systemic drug treatment and preventing the recurrence rate, there is scarce literature describing the use of PDT in this treatment, and correct protocols have yet to be established. We conducted a short- and long-term controlled clinical trial to evaluate and compare the efficacy of combined $\mathrm{TN}+\mathrm{MB} / \mathrm{PDT}$ versus $\mathrm{TN}+\mathrm{MAL} / \mathrm{PDT}$ in the treatment of severe toenail onychomycosis, as per clinical and mycological rates.

\section{MATERIALS AND METHODS}

\section{Study design}

A randomized, 52-week, single-blind, controlled trial was designed. Patients with severe toenail onychomycosis affecting the big toe, with mycological diagnosis of onychomycosis (nail biopsy: PAS stain $(+)$ and fungal culture for dermatophytes $(+))$, were recruited from the Private Clinic of Dr. Alberdi and randomly allocated to two different treatment groups by means of a computer-generated random list: group I: $\mathrm{TN}+\mathrm{MB} / \mathrm{PDT}$ or group II: $\mathrm{TN}+\mathrm{MAL} / \mathrm{PDT}$. Characteristics of the recruited patients such as kind of agent identified (dermatophyte infection), severity (OSI value $\sim 20$ ), involvement of the disease ( $>60 \%$ involvement of the nail matrix) and chronicity are shown in Table 1 . Normal results in a liver function test were also required. Exclusion criteria were: under 18 years of age, contraindications for the use of MB (glucose-6-phosphate dehydrogenase deficiency), contraindications for the use of MAL (porphyria diagnosis), suffering from any disease that can cause nail abnormalities and can interfere with evaluation (for example tinea pedis, atopic dermatitis, psoriasis or lichen planus), internal diseases, liver or kidney impairment, pregnancy or breastfeeding, use of topical or 
systemic antifungals within 0 to 24 weeks prior to enrollment in the trial and concomitant use of drugs that could interact with TN.

The study protocol was granted Ethics Committee approval by the Hospital Clínico San Carlos in Madrid, Spain (Internal code: 17/501-E) in accordance with the ethical principles declared by the World Medical Association of Helsinki (2013). ${ }^{21}$ All patients understood and signed an informed consent form before their inclusion in the study. They showed willingness and ability to comply with study instructions and commit to attending all visits.

Two weeks before treatment, all patients included in the study received pretreatment instructions: $40 \%$ urea to be applied regularly during research monitoring and 5-7 days before starting oral TN administration and PDT sessions. This treatment was complemented by nine PDT sessions over the course of 16 weeks. In parallel, clinical efficacy of treatment was assessed by digital photographs at baseline and each follow-up visit. Microbiological examination by mycological culture and specimen collection for histological examination (PAS stain) were carried out before the study and at 16, 40 and 52-week follow-up. Side effects were recorded during each treatment session. A schematic picture of the study protocol is shown in Figure 1.

\section{Study sample}

Sample size calculation by IBM SPSS SamplePower (v3.0) was based on detecting a difference between groups of 10 points or more in the primary outcome variable, Onychomycosis Severity Index (OSI), with the assumption of a common standard deviation (SD) of 6, error of 0.05 and power of $90 \%$. The sample size calculation indicated that we needed to enrol 20 patients at a 1:1 allocation ratio of $\mathrm{TN}+\mathrm{MB} / \mathrm{PDT}$ to $\mathrm{TN}+\mathrm{MAL} / \mathrm{PDT}$. Yet, a total of 24 patients (12 per group) were initially enrolled for better consideration and to compensate for loss to follow-up, based on previous pilot studies.

Participants were treated by a nonblinded researcher. All patients were evaluated by a blinded researcher (same researcher for all patients) at baseline, 16, 40 and 52-week follow-ups. 


\section{Treatment}

All selected patients received $250 \mathrm{mg} /$ day of oral TB for 12 weeks. The plates of the affected toenails were softened with $40 \%$ urea ointment applied every evening for 5-7 days before each PDT session to enhance PS penetration. Periungual skin was protected with Vaseline to avoid any lesion produced by the urea. The schedule of the combined treatment is shown in Figure1.

Group I protocol: Patients of this group combined oral treatment with nine sessions of MB/PDT (2\% methylene blue aqueous solution). MB solution was applied on the affected area for scarcely 3 min. as incubation time.

Group II protocol: Patients of this group combined oral treatment with nine sessions of MAL/PDT (Metvix ${ }^{\circledR}$, Galderma, La Defense Cedex, France). In each session, MAL was applied to the affected ungual area, covered with a plastic occlusive dressing and protected from light during $3 \mathrm{~h}$.

Irradiation was carried out in both groups after incubation using a $635 \mathrm{~nm}$ LED lamp (Aktilite ${ }^{\circledR}$, Photocure ASA, Oslo, Norway) at a fluence of $37 \mathrm{~J} / \mathrm{cm}^{2}$ during $10 \mathrm{~min}$, resulting in a total dose of approximately $62 \mathrm{~mW} / \mathrm{cm}^{2}$ per session. The lamp should be placed at a distance of $100 \mathrm{~mm}$ from the skin.

Side effects were recorded at each visit. Patients were also monitored for complete blood count and liver enzyme levels every 6 weeks due to the use of oral TN.

\section{Monitoring and assessment timeline}

Clinical efficacy determination

Clinical improvement was determined using the Onychomycosis Severity Index (OSI), obtained by scoring the photographs taken during the course of the study. OSI was obtained by multiplying the score for the affected area (range 0-5) by the score for the proximity of disease to the matrix (range 1-5), adding 10 points for longitudinal striae, patches (dermatophytoma) or subungual hyperkeratosis greater than $2 \mathrm{~mm}$. The result allows the classification of the 
condition as: mild (score of 1-5), moderate (6-15) or severe (16-35), and consequently registers the efficacy of the treatment over the examined period. ${ }^{22}$ Scores were calculated at baseline and at 16, 40 and 52-week follow-up. Clinical cure was defined as a completely normal appearance of the nail or the presence of $\leq 5 \%$ nail plate involvement in onychomycosis.

Following the same criteria described by Tatawy et al, ${ }^{23}$ the difference between OSI score at baseline and that obtained at 16, 40 and 52-week follow-up was calculated and transformed into percentage change in OSI score from baseline. In this way, the values were classified as follows: i) $0-25 \%$ (no improvement), ii) $26-50 \%$ (mild improvement), iii) $51-75 \%$ (moderate improvement) and iv) 76-100\% (market improvement) (see Table 2).

Mycological efficacy determination

Microbiological samples for performing fungal cultures and affected-nail samples for histological analysis (PAS diastase stain) were obtained before the initiation of the study and at 16,40 and 52-week follow-up.

Complete cure

Complete cure was defined as clinical cure and mycological cure (MC).

Treatment success

Treatment success was defined as MC plus less than $10 \%$ of nail affected.

Clinical improvement

Clinical improvement was defined as MC plus less than $25 \%$ of nail affected.

\section{Statistical analysis}

Statistical analysis was performed using a SPSS software package for Windows (version 25.0; SPSS, Chicago, III). Once the normality of the studied variables (OSI, degree of improvement, $\%$ of involvement and mycological cure evolution) was verified via the Shapiro-Wilk test, the variables were analyzed by two-factor repeated measures ANOVA (intrasubject factor (time) 
and intersubject factor (procedure)) with Post hoc Bonferroni correction for intragroup comparison. A $p$-value of $<0.05$ was considered statistically significant.

\section{RESULTS}

After applying restrictive inclusion and exclusion criteria and accounting for dropouts and the exclusion of patients with incompletely recorded histories, the final study population consisted of 20 subjects (12 men and 8 women). 10 patients constituted each group. Group I

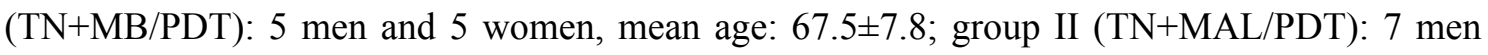
and 3 women, mean age: $66.4 \pm 16.7$. Demographic data of both groups are shown in Table 1 .

The results obtained in the present study showed clinical and mycological improvement of the target nails during treatment, mainly at the end of the evaluated period of time ( $\sim 52$ weeks from baseline or nine months after completing PDT sessions). Complications or side effects were not observed during or after any of the TN+PDT combination modalities, and the level of patient satisfaction was positive. TN was very well tolerated. Only two patients within group I reported gastrointestinal discomfort due to the continuous oral treatment with TN (but this did not prevent them from continuing with the treatment). As for group II, some patients referred pain sensation or itching during irradiation time, probably due to the skin coming into contact with MAL as a result of an accidental displacement of the occlusive dressing during the incubation period.

Figure 2 and 3 show the obvious clinical responses, at 52-week follow-up compared to baseline, in toenails after $\mathrm{TN}+\mathrm{MB} / \mathrm{PDT}$ (Figure 2) and $\mathrm{TN}+\mathrm{MAL} / \mathrm{PDT}$ (Figure 3), respectively. Clinical cure rates increased from the start of the treatment to the 52-week follow-up, mainly during the last third of the study. Both protocols significantly reduced OSI scores $(p<0.05)$ over the evaluated period of time, from $24.2 \pm 4.6$ to $0.7 \pm 0.6$ (group I)) and from $18.5 \pm 10.1$ to $2.1 \pm 2.0$ (group II) (Table 2), a little faster in group I, but not statistically significant between the groups $(\mathrm{p}<0.05)$. Table 2 also shows the degree of improvement during the course of the study. Its 
evolution was statistically significant within each group, from $49.6 \pm 19.5$ to $97.6 \pm 3.4$ (group I)) and from $60.8 \pm 37.6$ to $89.2 \pm 25.8$ (group II) but without significant intergroup differences. In addition, the percentage of nail involvement decreased significantly during the study in both groups, from $64.8 \pm 24.3$ to $1.7 \pm 1.6$ (group I)) and from $63.6 \pm 41.6$ to $6.0 \pm 5.5$ (group II) (Table 2), both modalities following a very similar trend throughout the study.

The evolution of the mycological response by histological examination over the analyzed period (until 52 weeks) is shown in Table 2 and Figure 4. Infection diminished during treatment; both modalities showed a significant decrease during the last third of the evaluated period, both following a similar trend. Figure 4 shows the different cure rates obtained at the different time points. The two modalities showed similar positive outcomes at the end of the treatment, when the nails had almost completely regrown. The nonexistence of significant differences between group I and group II could be explained by the potent action of TN as first-line therapy, common in both combinations.

\section{DISCUSSION}

In onychomycosis it is important to confirm the clinical diagnosis by mycological laboratory methods before initiating systemic antifungal treatment, especially as antifungal sensitivity and susceptibility may differ between different genera and species. ${ }^{24}$ In this way, mycological diagnosis may guide the appropriate choice of antifungal treatment. In addition, the viability of the infecting organism must be assessed once treatment stops, in order to decide whether to prolong or change the antimycotic treatment. In the inclusion criteria, we only selected patients with dermatophyte toenail onychomycosis, as it has been demonstrated that TN is ineffective for treating onychomycosis caused by nondermatophyte..$^{25}$

When onychomycosis affects the toenail matrix region, this infection is considered to be the most difficult to cure, given that the involvement of the most proximal part of the nail may affect the metabolism and growth of the nail. ${ }^{26}$ When percentage of toenail involvement is 
greater than $60 \%$, optimum treatment is based on oral TN plus topical therapy, ${ }^{4}$ restricting the use of topical therapy with Amorolfine, Ciclopirox, Tavaborole and Efinaconazole when nail involvement is $<60 \%$ (mild-moderate severity). ${ }^{27}$ Although dermatophytes are normally susceptible to $\mathrm{TN}$, onychomycosis can result in refractory chronic disease associated with the formation of biofilms. ${ }^{28}$ Recent photodynamic approaches hold great promise for combating the biofilm of dermatophytes involved in onychomycosis. ${ }^{28}$ In this context, PDT applied alongside antifungal agents is likely to reduce the duration and toxicity of the treatment and increase its efficacy.

This study evaluated the efficacy and safety of TN+MB/PDT vs. TN+MAL/PDT. Both PDT modalities were well tolerated, although some patients treated with MAL/PDT reported blistering of the nail bed, burning sensations and significant pain, effects that have already been mentioned in previous studies. ${ }^{14}$ Interestingly, a recent Cochrane review found that the risk of adverse events between $\mathrm{TN}$ and placebo was not statistically significant $(\mathrm{RR}=1.13 ; 95 \% \mathrm{CI}$, 0.87 to 1.47 ), indicating that oral $\mathrm{TN}$ treatment is generally safe. ${ }^{7}$

The average growth rate for toenails is 0.1 millimeters per day, or 3 millimeters per month. A toenail will therefore take 12 to 18 months to grow back. However, toenails grow slower as we age $(0.06 \mathrm{~mm} /$ day). Most published clinical trials on $\mathrm{TN}$ do not present results according to nail involvement or the patient's age. Furthermore, in these studies the dates of the last followups $(10,12,18$ months ...) do not coincide, which makes it difficult to make an exact comparison. Previous studies on the effects of continuous oral TN treatment showing a high degree of coincidence with the initial state of our patients include those of Gupta, ${ }^{29,30}$ Warshaw, ${ }^{31}$ Auvinen ${ }^{32}$, Elewski ${ }^{33}$, De Backer ${ }^{34}$ and Sigurgeirsson. ${ }^{35}$ Gupta et al. (nail involvement 67.6 $\%$, mean age 57.0 ) showed at 48 -week follow-up $71 \%$ of $\mathrm{MC}$ and $51.6 \%$ of effective cure (MC+ less than $10 \%$ of nail affected). ${ }^{29}$ The same author in another study with more elderly patients (nail involvement $67.5 \%$, mean age 68.0) obtained at 18-months follow-up 64\% of MC and $62.0 \%$ of clinical efficacy. ${ }^{30}$ Warshaw et al. (mean age $\sim 65.2$, nail involvement $\sim 86.7 \%$ ) reported at 18 -month follow-up $71 \%$ of MC and $40 \%$ of complete cure. ${ }^{31}$ Auvinen et al. (mean 
age $\sim 64$ ) obtained at 10 -month follow-up $56 \%$ of $\mathrm{MC}$ and $16 \%$ of complete cure. ${ }^{32}$ Elewski et al. (mean age $\sim 50.1$, nail involvement $\sim 48.7 \%$ ) showed at 48 -week follow-up $71.4 \%$ of MC and $37.1 \%$ of complete cure. ${ }^{33}$ De Backer et al. reported $73 \%$ of $\mathrm{MC}$ and $37.7 \%$ of complete cure at 48 weeks in patients over 18 years ${ }^{34}$ and Sigurgeirsson et al. (mean age $\sim 50.1$, nail involvement 73.7\%) obtained at 72-week follow-up 75.7\% of MC and $45.8 \%$ of complete cure. ${ }^{35}$ Results of the present study show $40 \%$ of MC at 40 -week follow-up. This rate is initially low given the almost total involvement of the nail by onychomycosis. At 52-week follow-up, the percentage of MC reached $\sim 95 \%$ and $\sim 70 \%$ of complete cure. Thus, the adjunctive treatment with PDT in any of the modalities tested here complements the effective action of TN.

Most studies with PDT use a treatment protocol of 2 sessions. As the number of sessions increases or the intervals between them are shortened or the energy/power of the applied light is intensified, the success rate will likely rise. ${ }^{36}$ We applied 9 sessions in order to increase the efficacy and prevent easy recurrence, especially common in the case of dermatophyte infections with a high involvement of the disease in the nail. Published clinical trials have reported on the use of MB/PDT after 12 sessions with a two-week interval between sessions, ${ }^{17,} 37$ in contrast with the 2-3 sessions (once a week) that are usually established for MAL/PDT. ${ }^{19}$ Our results showed similarity between the two treatment modalities after 9 sessions and improved cure rates compared with data published after treatment with TN alone. These results seem to indicate that, although the systemic action of TN is predominant over the topical action of PDT, the latter (with both MB and MAL) reinforces the antifungal action of TN.

Before pretreatment with $40 \%$ urea, mechanical removal of nail residue and hyperkeratotic areas was carried out to favor the penetration of MB/MAL across the infected area. ${ }^{19}$ At higher concentrations $(>10 \%)$, urea exerts an emollient/keratolytic action by breaking hydrogen bonds thus dissolving keratin. ${ }^{38}$ Therefore, urea pretreatment softens the nail plate and favors hyperkeratosis removal and the reduction of nail plate thickness, enhancing the LED light penetration through the nail plate in order to reach the fungal colonies of the nail bed and nail matrix, thereby improving the clinical response to PDT. ${ }^{13,39}$ Consequently, this pretreatment can 
also minimize incubation time for MB/MAL by enhancing PS penetration and making the treatment more effective. Even so, there is difference between the incubation times for MB and MAL (3 min and $3 \mathrm{~h}$, respectively). As soon as it is applied, MB diffuses through the nail and its photoactivation is immediate. MAL is not in itself a photosensitizer and it needs to be previously metabolized inside the cells to protoporphyrin IX which is the photoactive compound.

OSI provides a standardized method for evaluating onychomycosis that can be utilized throughout a patient's treatment course. This is a promising tool; however, further research is necessary in order to properly correlate nail disease severity with prognostic outcomes. Mycological cure is also another common outcome measure. After treatment has ended, fungal cells can remain viable in the nail bed, regrow and start a new infection. ${ }^{14,40}$ Due to its anatomic complexity, the nail bed shows poor vascularization, hindering the entry of immune cells or systemic antifungal drugs. This results in slower healing and increased risk for recurrence. ${ }^{41}$ This complexity also hinders the removal of this type of infection. In our study, there were no cases of recurrence during the 52-week observation period.

Regarding combined treatments of TN with another agent, some studies have evaluated the combination with topical Ciclopirox, concluding that the combination therapy of oral TN and Ciclopirox nail lacquer is a safe and more effective treatment for onychomycosis than $\mathrm{TN}$ alone, ${ }^{42}$ especially in younger patients and in shorter-duration onychomycosis. ${ }^{43}$ There are also studies with Amorolfine showing that in the treatment of toenail onychomycosis with matrix involvement, Amorolfine nail lacquer in combination with oral TN enhances clinical efficacy and is more cost-effective than TN alone.${ }^{44}$ However, there are no published studies where TN is combined with PDT, ours being the pioneer. In our study, both MB/PDT and MAL/PDT show properties that could perform well in combination with TN. While TN reaches the nail via the nail bed by diffusion from dermal vessels and via the matrix by incorporation into the growing onychocytes, PDT reaches the lateral edge of the nail, complementing and improving the effects of TN. Thus, our study concludes that MB/PDT and MAL/PDT have a synergistic 
effect when used in combination with $\mathrm{TN}$ in the treatment of severe toenail onychomycosis with matrix involvement.

Studies suggest that porphyrin prodrugs (for example MAL) generally cause oxidative damage via the reactive singlet oxygen $\left({ }^{1} \mathrm{O}_{2}\right)$, causing fungal membrane disruption and mitochondrial damage (PpIX is here synthesized) but not DNA damage. ${ }^{45,46} \mathrm{MB}$ is known to localize in the fungal membrane. Consequently, this is the cellular structure damaged upon illumination, resulting in an increased permeability which leads to cell death. ${ }^{47}$ DNA damage is also reported. ${ }^{48}$ For both MB/PDT and MAL/PDT, absence of genotoxic and mutagenic effects on both fungal cells and keratinocytes seems a fundamental premise for the long-term safety of the treatment, e.g., the lack of selection of drug-resistant fungal strains and cancerogenesis. ${ }^{49}$ As for the TN mechanism, this active antimycotic agent inhibits squalene epoxidase which blocks the biosynthesis of ergosterol, the main component of fungal cell membranes which has fungistatic and fungicidal actions. ${ }^{9}$ Finally, it should be noted that photobiomodulation for MB/MAL photoexcitation reduces treatment time and enhances the appearance of nails contaminated with onychomycosis. ${ }^{3}$

To date, it is still difficult to compare the results of different clinical trials based on the use of PDT in onychomycosis due to variations in the causal agent, differences in percentage of nail involvement with or without the combination with oral systemic drugs; differences in factors associated with the technical protocol, such as the photosensitizer used, number of sessions administered, characteristics of irradiation source and pretreatment mode (urea, fractional laser radiation). Light therapy is a promising adjunctive method to shorten the conventional treatment or improve the poor response expected in nails with high percentage of onychomycosis involvement.

This is a pilot study comparing the efficacy of two combined treatments, which have never been compared, hence their novelty. Thus, further investigation based in the comparison of these combination treatment modalities with conventional topical and oral antifungal treatment are 
necessary in order to delimitate their power and effectiveness. Our results show that either PDT modality used here accelerates the healing process of the oral TN treatment. Although the sample size may not have been large enough to detect statistical significance, this study provides the basis for longer studies that evaluate the efficacy of other protocols based on the combination of PDT and TN. A long-term study (beyond 18 months) with a larger sample size could provide additional evidence and ultimately elucidate the final effects of both combination treatments on recrudescence. 


\section{REFERENCES}

1.- Hay R. Literature review. Onychomycosis. J Eur Acad Dermatol Venereol. 2005; 19 (Suppl 1): $1-7$.

2.-Faergemann J, Baran R. Epidemiology, clinical presentation and diagnosis of onychomycosis. Br J Dermatol. 2003; 149 (Suppl 65): 1-4.

3.- Finch JJ, Warshaw EM. Toenail onychomycosis: current and future treatment options. Dermatol Ther. 2007; 20(1): 31-46.

4.- Hanna S, Andriessen A, Beecker J, Gilbert M, Goldstein E, Kalia S, King A, Kraft J, Lynde C, Singh D, Turchin I, Zip C. Clinical insights about onychomycosis and its treatment: a consensus. J Drugs Dermatol. 2018; 17(3):253-262.

5.- Burzykowski T, Molenberghs G, Abeck D, Haneke E, Hay R, Katsambas A, Roseeuw D, van de Kerkhof P, van Aelst R, Marynissen G. High prevalence of foot diseases in Europe: results of the Achilles Project. Mycoses 2003; 46 (11-12):496-505.

6.- Roberts DT, Taylor WD, Boyle J. Guidelines for treatment of onychomycosis. $\mathrm{Br} J$ Dermatol. 2003; 148(3): 402-410.

7.- Kreijkamp-Kaspers S, Hawke K, Guo L, Kerin G, Bell-Syer SE, Magin P, Bell-Syer SV, van Driel ML. Oral antifungal medication for toenail onychomycosis. Cochrane Database Syst Rev. 2017, Issue 7. Art. No: CD010031. Doi: 10.1002/14651858.CD010031.pub2.

8.- Gupta AK, Ryder JE, Johnson AM. Cumulative meta-analysis of systemic antifungal agents for the treatment of onychomycosis. Br J Dermatol. 2004; 150(3): 537-544.

9.- Lamisil (terbinafine hydrochloride tablets) Prescribing information. [WWW document]. Novartis Pharmaceuticals Corporation, East Hanover, N], USA, URL http://www.pharma.us.novartis.com/product/pi/pdf/Lamisil tablets.pdf [accessed on 20 December 2011].

10.- Waibel J, Wulkan AJ, Rudnick A. Prospective efficacy and safety evaluation of laser treatments with real-time temperature feedback for fungal onychomycosis. J Drugs Dermatol. 2013; 12(11): 1237-1242.

11.- Kimura U, Takeuchi K, Kinoshita A, Takamori K, Hiruma M, Suga Y. Treating onychomycoses of the toenail: clinical efficacy of the sub-millisecond 1,064 nm Nd:YAG laser using a 5 mm spot diameter. J Drugs Dermatol. 2012; 11(4): 496-504.

12.- Alberdi, E, Gómez C. Efficiency of methylene blue mediated photodynamic therapy vs intense pulsed light in the treatment of onychomycosis in the toenails. Photodermatol Photoimmunol Photomed. 2019; 35(2): 69-77.

13.- Piraccini BM, Rech G, Tosti A. Photodynamic therapy of onychomycosis caused by Trichophyton rubrum. J Am Acad Dermatol. 2008; 59 (5 Suppl): S75-S76.

14.- Sotiriou E, Koussidou-Eremonti T, Chaidemenos G, Apalla Z, Ioannides D. Photodynamic therapy for distal and lateral subungual toenail onychomycosis caused by Trichophyton rubrum: preliminary results of a single-centre open trial. Acta Derm Venereol. 2010; 90(2): 216-217. 
15.- Oleinick NL, Morris RL, Belinchenko I. The role of apoptosis in response to photodynamic therapy: what, were, why and how. Photochem Photobiol Sci. 2002; 1(1): 1-21.

16.- Dai T, Fuchs BB, Coleman JJ, Prates RA, Astrakas C, St Denis TG, Ribeiro MS, Mylonakis E, Hamblin MR, Tegos GP. Concepts and principles of photodynamic therapy as an alternative antifungal discovery platform. Front Microbiol. 2012; 3:120.

17.- Figueiredo Souza LW, Souza SV, Botelho AC. Randomized controlled trial comparing photodynamic therapy base on methylene blue dye and fluconazole for toenail onychomycosis. Dermatol Ther. 2014; 27(1): 43-47.

18.- Kamp H, Tietz HJ, Lutz M, Piazena H, Sowyrda P, Lademann J, Blume-Peytavi U. Antifungal effect of 5-aminolevulinic acid PDT in Trichophyton rubrum. Mycoses 2005; 48(2):101-107.

19.- Gilaberte Y, Robres MP, Frías MP, García-Doval I, Rezusta A, Aspiroz C. Methyl aminolevulinate photodynamic therapy for onychomycosis: a multicentre, randomized, controlled clinical trial. J Eur Acad Dermatol Venereol. 2017; 31(2): 347-354.

20.- Robres P, Aspiroz C, Rezusta A, Gilaberte Y. Usefulness of photodynamic therapy in the management of onicomicosis. Actas Dermosifiliogr. 2015; 106 (10):795-805.

21.- World Medical Association. World Medical Association Declaration of Helsinki: Ethical principles for medical research involving human subjects. JAMA. 2013; 310(20):2191-2194.

22.- Carney C, Tosti A, Daniel R, Scher R, Rich P, DeCoster J, Elewski B. A new classification system for grading the severity of onychomycosis: Onychomycosis severity index. Arch Dermatol. 2011; 147(11):1277-1282.

23.- El-Tatawy RA, Aliweh HA, Hegab DS, Talaat RAZ, Shams Eldeen MA. Fractional carbon dioxide laser and topical tioconazole in the treatment of fingernail onychomycosis. Lasers Med Sci. 2019; 34(9):1873-1880.

24.- Gupta AK, Versteeg SG, Shear NH. Confirmatory testing prior to initiating onychomycosis therapy is cost-effective. J Cutan Med Surg. 2018; 22 (2):129-141.

25.- Baudraz-Rosselet F, Ruffieux C, Lurati M, Bontems O, Monod M. Onychomycosis insensitive to systemic terbinafine and azole treatments reveals non-dermatophyte moulds as infectious agents. Dermatology. 2010; 220 (2): 164-168.

26.- Baran R, Feuilhade M, Combernale P, Datry A, Goettmann S, Pietrini P, Viguie C, Badillet G, Larnier C, Czernielewski J. A randomized trial of amorolfine 5\% solution nail lacquer combined with oral terbinafine compared with terbinafine alone in the treatment of dermatophytic toenail onychomycoses affecting the matrix region. Br J Dermatol. 2000; 142 (6):1177-1183.

27.- Gupta AK, Daigle D, Foley KA. Topical therapy for toenail onychomycosis: an evidencebased review. Am J Clin Dermatol. 2014; 15(6): 489-502.

28.- Chen B, Sun Y, Zhang J, Chen R, Zhong X, Wu X, Zheng L, Zhao J. In vitro evaluation of photodynamic effects against biofilms of dermatophytes involved in Onychomycosis. Front Microbiol. 2019; 10:1228. 
29.- Gupta AK, Lynch LE, Kogan N, Cooper EA. The use of an intermittent terbinafine regimen for the treatment of dermatophyte toenail onychomycosis. $J$ Eur Acad Dermatol Venereol. $2009 ; 23(3), 256-262$.

30.- Gupta AK, Konnikov MD, Lynde CW. Single-blind, randomized, prospective study on terbinafine and itraconazole for treatment of dermatophyte toenail onychomycosis in the elderly. J Am Acad Dermatol. 2001; 44: 479-484.

31.- Warshaw EM, Fett DD, Bloomfield HE, Grill JP, Nelson DB, Quintero V, Carver SM, Zielke GR, Lederle FA. Pulse versus continuous terbinafine for onychomycosis: a randomized, double-blind, controlled trial. J Am Acad Dermatol. 2005; 53(4):578-584.

32.- Auvinen T, Tiihonen R, Soini M, Wangel M, Sipponen A, Jokinen JJ. Efficacy of topical resin lacquer, amorolfine and oral terbinafine for treating toenail onychomycosis: a prospective, randomized, controlled, investigator-blinded, parallel-group clinical trial. Br J Dermatol. 2015; 173(4):940-948.

33.- Elewski B, Pollak R, Ashton S, Rich P, Schlessinger J, Tavakkol A. A randomized, placebo- and active-controlled, parallel-group, multicentre, investigator-blinded study of four treatment regimens of posaconazole in adults with toenail onychomycosis. Br J Dermatol. 2012; 166(2):389-398.

34.- De Backer M, De Vroey C, Lesaffre E, Scheys I, De Keyser P. Twelve weeks of continuous oral Therapy for toenail onychomycosis caused by dermatophytes: A double-blind comparative trial of terbinafine $250 \mathrm{mg}$ /day versus itraconazole $200 \mathrm{mg} / \mathrm{day}$. J Am Acad Dermatol. 1998; 38 (5Pt3): S57-S63.

35.- Sigurgeirsson B, Billstein S, Rantanen T, Ruzicka T, Di Fonzo E, Vermeer BJ, Goodfield MJD, Evans EGV, for the L.I.ON. study group. L.I.ON. Study: efficacy and tolerability of continuous terbinafine $\left(\right.$ Lamisil $\left.^{\circledR}\right)$ compared to intermittent itraconazole in the treatment of toenail onychomycosis. Br J Dermatol. 1999; 141 (Suppl. 56): 5-14.

36.- Sabbah L, Gagnon C, Bernier FE, Maari C. A randomized, double-blind, controlled trial evaluating the efficacy of Nd:YAG $1064 \mathrm{~nm}$ short-pulse laser compared with placebo in the treatment of toenail onychomycosis. J Cut Med Surg. 2019; 23(5):507-512.

37.- Souza LW, Souza SV, Botelho AC. Distal and lateral toenail onychomycosis caused by Trichophyton rubrum: treatment with photodynamic therapy based on methylene blue dye. $A n$ Bras Dermatol. 2014; 89(1): 184-186.

38.- Pan M, Heinecke G, Bernardo S, Tsui C, Levitt J. Urea: A comprehensive review of the clinical literature. Dermatol Online J. 2013; 19(11): 20392.

39.- Kalokauidis K, Onder M, Trakatelli MG, Richert B, Fritz K. The effect of Q-switched Nd:YAG $1064 \mathrm{~nm} / 532 \mathrm{~nm}$ laser in the treatment of onychomycosis in vivo. Dermatol Res Pract. 2013; 2013: 379725 .

40.- Morgado LF, Trávolo ARF, Muehlmann LA, Narcizo PS, Nunes RB, Pereira PAG, PyDaniel KR, Jiang CS, Gu J, Azevedo RB, Longo JPF. Photodynamic therapy treatment of onychomycosis with Aluminium-Phthalocyanine Chloride nanoemulsions: a proof of concept clinical trial. J Photochem Photobiol B. 2017; 173: 266-270. 
41.- Piraccini BM, Sisti A, Tosti A. Long-term follow-up of toenail onychomycosis caused by dermatophytes after successful treatment with systemic antifungal agents. $J$ Am Acad Dermatol. 2010; 62(3): 411-414.

42.- Gupta AK, Onychomycosis Combination Therapy Study Group. Ciclopirox topical solution $8 \%$ combined with oral terbinafine to treat onychomycosis: a randomized, evaluator-blinded study. J Drugs Dermatol. 2005; 4(4):481-485.

43.- Avner S, Nir N, Henri T. Combination of oral terbinafine and topical ciclopirox compared to oral terbinafine for the treatment of onychomycosis. J Dermatolog Treat. 2005; 16(5-6):327330 .

44.- Baran R, Sigurgeirsson B, de Berker D, Kaufmann R, Lecha M, Faergemann J, Kerrouche N, Sidou F. A multicentre, randomized, controlled study of the efficacy, safety and costeffectiveness of a combination therapy with amorolfine nail lacquer and oral terbinafine compared with oral terbinafine alone for the treatment of onychomycosis with matrix involvement. Br J Dermatol. 2007; 157(1):149-157.

45.- Hsieh YS, Wu CC, Chang CJ, Yu JS. Subcellular localization of Photofrin determines the death phenotype of human epidermoid carcinoma A431 cells triggered by photodynamic therapy: when plasma membranes are the main targets. J Cell Physiol. 2003; 194(3):363-375.

46.- Lambrechts SA, Aalders MC, Van Marle J. Mechanistic study of the photodynamic inactivation of Candida albicans by a cationic porphyrin. Antimicrob Agents Chemother. 2005; 49(5):2026-2034.

47.- Donnelly RF, McCarron PA, Tunney MM. Antifungal photodynamic therapy. Microbiol Res. 2008; 163(1): 1-12.

48.- Berra CM, de Oliveira CS, Garcia CC, Rocha CR, Lerner LK, Lima LC, Baptista Mda S, Menck CF. Nucleotide excision repair activity on DNA damage induced by photoactivated methylene blue. Free Radic Biol Med. 2013; 61: 343-356.

49.- Calzavara-Pinton PG, Venturini M, Sala R. A comprehensive overview of photodynamic therapy in the treatment of superficial fungal infections of the skin. $J$ Photochem Photobiol $B$. 2005; 78 (1): 1-6. 
Table 1.- Clinical characteristics of recruited patients.

\begin{tabular}{|l|l|l|}
\cline { 2 - 3 } \multicolumn{1}{l|}{} & TN+MB/PDT & TN+MAL/PDT \\
\hline Sex & & $7(70 \%)$ \\
Male [n (\%)] & $5(50 \%)$ & $3(30 \%)$ \\
Female [n (\%)] & $5(50 \%)$ & $66.4 \pm 16.7 ;[33-84] ; 73$ \\
Age [mean $\pm(\mathrm{SD})]$; range; median & $67.5 \pm 7.8 ;[55-78] ; 69$ & \\
\hline Hystological analysis & $10(100 \%)$ & $10(100 \%)$ \\
PAS stain + & $0(0 \%)$ & $0(0 \%)$ \\
PAS stain - & & \\
\hline Microbiological analysis & $9(90 \%)$ & $8(80 \%)$ \\
T rubrum & $1(0 \%)$ & $2(20 \%)$ \\
T mentagrophytes & $24.2 \pm 4.6$ & $18.5 \pm 10.1$ \\
\hline Baseline OSI [mean $\pm(\mathrm{SD})]$ & & \\
\hline Chronicity & 3 & 4 \\
$<3$ years & 6 & 2 \\
3-5 years & 2 & 4 \\
$>5$ years & $64.8 \pm 24.3$ & $63.6 \pm 41.6$ \\
\hline Nail involvement $(\%)$ & &
\end{tabular}


Table 2.- Evolution of the Onychomycosis Severity Index, the degree of improvement, the percentage of disease involvement and the mycological response.

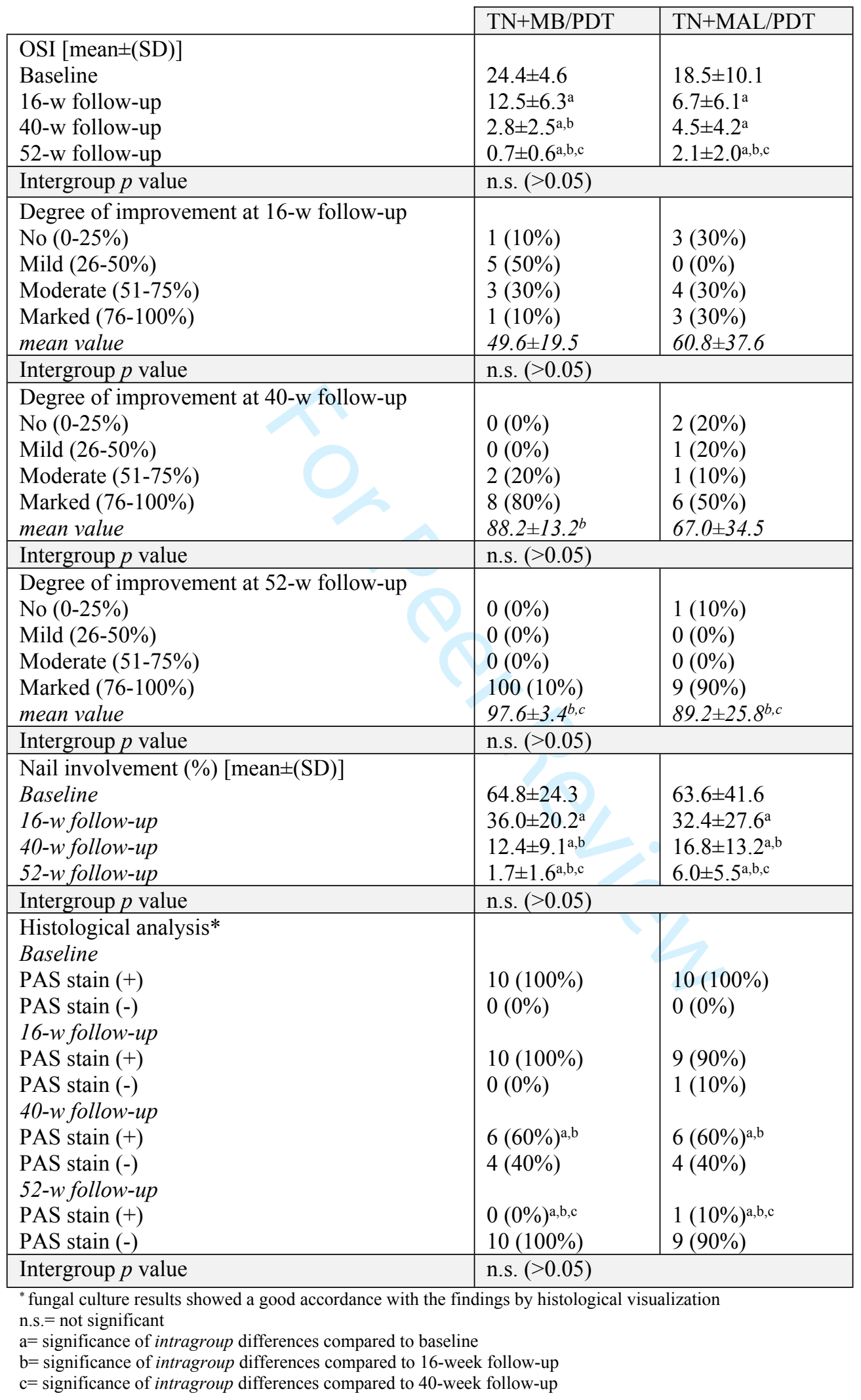




\section{FIGURE LEGENDS}

Figure 1.- Graphical representation of the clinical trial timeline and the timepoints used for experimental interventions and clinical and microbiological assessment.

Figure 2.- Clinical evolution of two toenail treated by $\mathrm{TN}+\mathrm{MB} / \mathrm{PDT}$ assessed by photographs: A) baseline; B) 52-week follow-up.

Figure 3.- Clinical evolution of two toenail treated by TN+MAL/PDT assessed by photographs: A) baseline; B) 52-week follow-up.

Figure 4.- Cure rates at 16, 40 and 52-week follow-ups. 


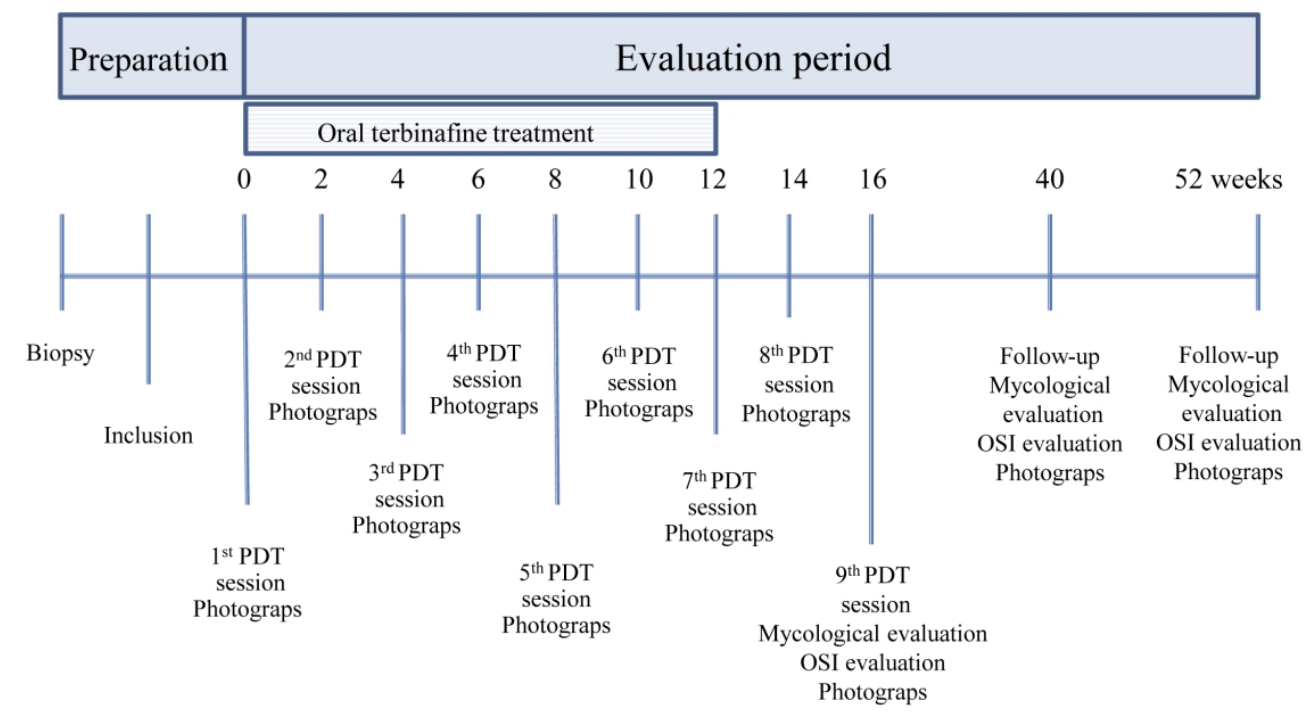

Figure 1

Figure 1.- Graphical representation of the clinical trial timeline and the timepoints used for experimental interventions and clinical and microbiological assessment. 

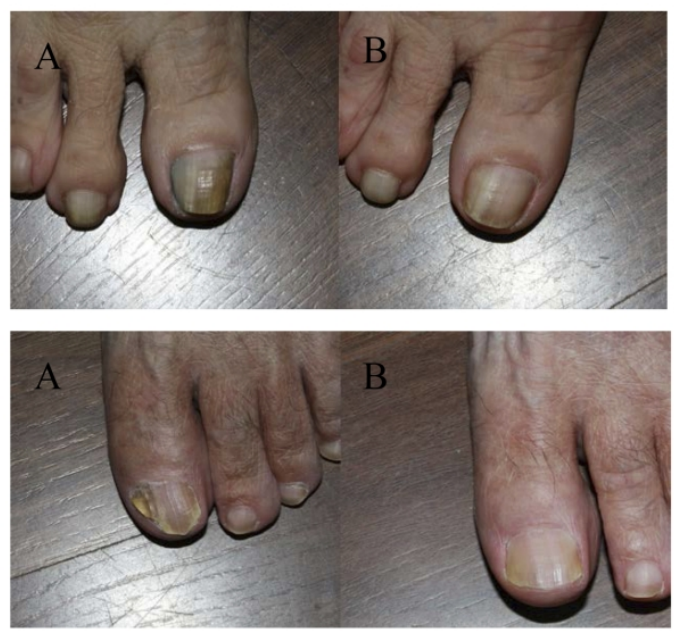

Figure 2

Figure 2.- Clinical evolution of two toenail treated by TN+MB/PDT assessed by photographs: A) baseline; B) 52-week follow-up. 

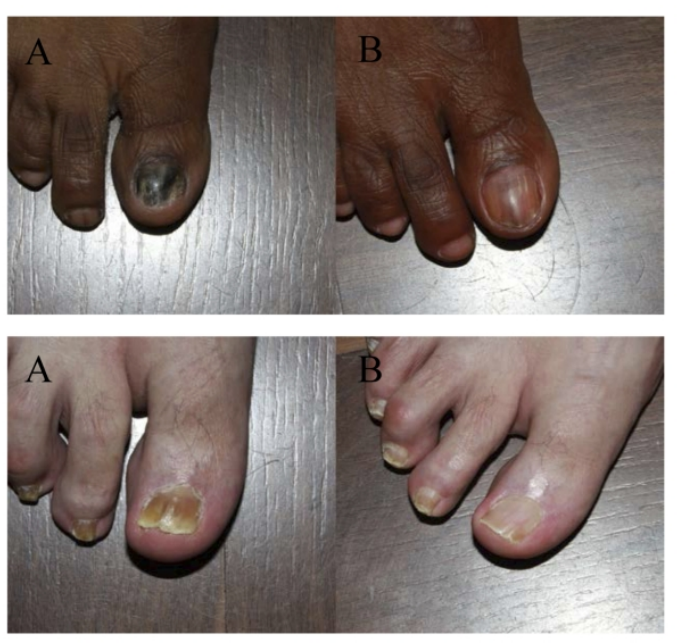

Figure 3

Figure 3.- Clinical evolution of two toenail treated by TN+MAL/PDT assessed by photographs: A) baseline; B) 52-week follow-up. 


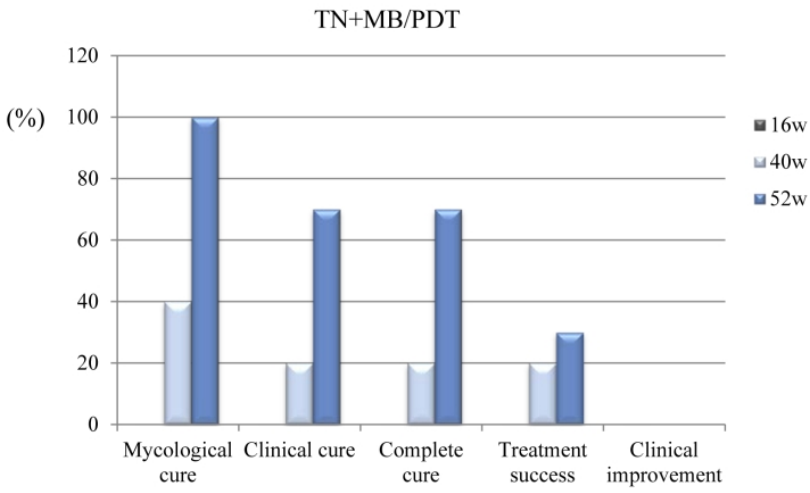

$\mathrm{TN}+\mathrm{MAL} / \mathrm{PDT}$

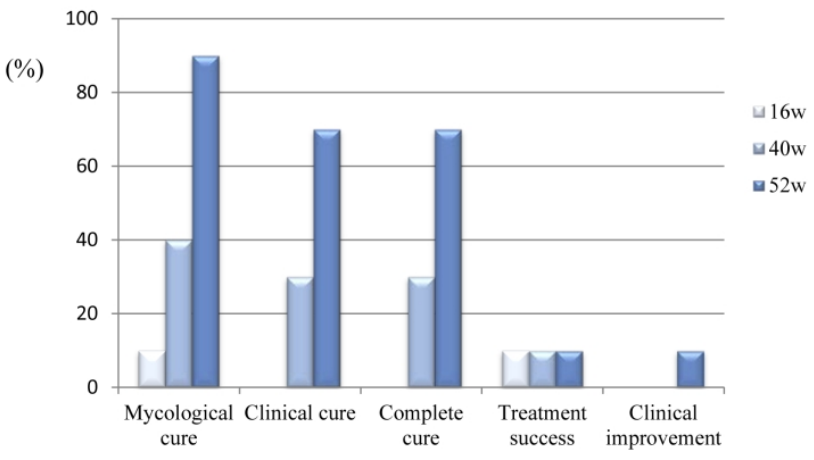

Figure 4

Figure 4.- Cure rates at 16, 40 and 52-week follow-ups. 